\title{
Retrieved Optical Thickness for Analyzing The Aerosol and Cloud Properties Using Lidar Remote Sensing
}

\author{
Syamsir Dewang \\ Physics Department, Faculty of Mathematics and Natural Sciences \\ Hasanuddin University, Makassar
}

Diterima 24-10-2009 Disetujui 14-05-2010

\begin{abstract}
The lidar remote sensing is the one important application to observe the aerosol and cloud of the atmosphere. The micropulse lidar (MPL) return signals were studied in the tropical area. In this investigation, the single scattering is analyzed by the physical properties of aerosol and cloud. The signal simulation of the single scattering predicts the maximum optical thickness by saturation. It was observed that saturation optical thickness from the lidar signal depends on the variation of extinction coefficient. This simulation is compared by the optical thickness estimation from the lidar data. The MPL data (at wavelength of $523 \mathrm{~nm}$ ) was determined, and the sky radiometer (at wavelength $500 \mathrm{~nm}$ ) was used as reference data. The maximum optical thickness of lidar was 2.6 at night time, and the maximum optical depth of lidar and sky radiometer data on the same day were 2.25 and 1.7, respectively.
\end{abstract}

Keywords: aerosol and cloud, extinction coefficient, micro pulse lidar (MPL), optical thickness

\section{INTRODUCTION}

The aerosol particles affect the climate directly by interacting with solar and terrestrial radiation and indirectly by their affects on cloud microphysics, and precipitation. Biomas burning is a major source not only trace gas emission into the troposphere but also organic hygroscopic particles. These smoke particles deteriorate the local ground visibility and give rise to increase the aerosol with smaller geometric radius over a relatively long period. (Shiina, 2005).

Lidar (light detection and ranging) is one application of the optical technology. It is an important tool for detecting physical aspect of aerosol and cloud, and for directly providing their height and thickness. The lidar sensing is affected by two physical parameters, i.e. extinction and backscattering coefficient. The application of lidar is used in environmental remote sensing to observe the air pollution, which is caused by the industry, and the forest fire. Another application is the observation of physical characteristic of aerosol and cloud such as height and size distribution, temperature, velocity of wind, extinction coefficient, and optical thickness. (Niranjan, 2007).
This research investigates the single scattering process to analyze the optical properties of aerosol and cloud such as optical thickness at boundary layer and high altitude cloud. In the first section, the saturation of optical thickness from lidar signal is simulated to estimate the maximum optical thickness. The saturation depends on both variation of extinction coefficient of the cloud, and cloud physical thickness. The second part is the estimation of optical thickness using micro pulse lidar (MPL), and there were compared by sky radiometer as reference data. The optical thickness is derived by integration of the extinction coefficient obtained by the Fernald equation (Lottman, 2001).

Theoryote. The lidar system is one of the tools for detecting many aspects of atmosphere. In general, a lidar system consist of 3 parts, i.e, laser source, receiver (telescope) and signal processing, which is include the photo-multiplier-tube (PMT) and display. The laser light source produces light shots directed to atmosphere. The transmitted signal induces the backscattering process from the aerosol and and clouds. Thus the backscattering signal can be detected by telescope. Furthermore, the geometrical form factor, $G(r)$ includes the degree of overlap between the laser beam and the field of view (FOV) of telescope. The 
simple lidar system is shown in Figure 1. The power received by the telescope may be expressed by lidar equation (Dewang, 2000).

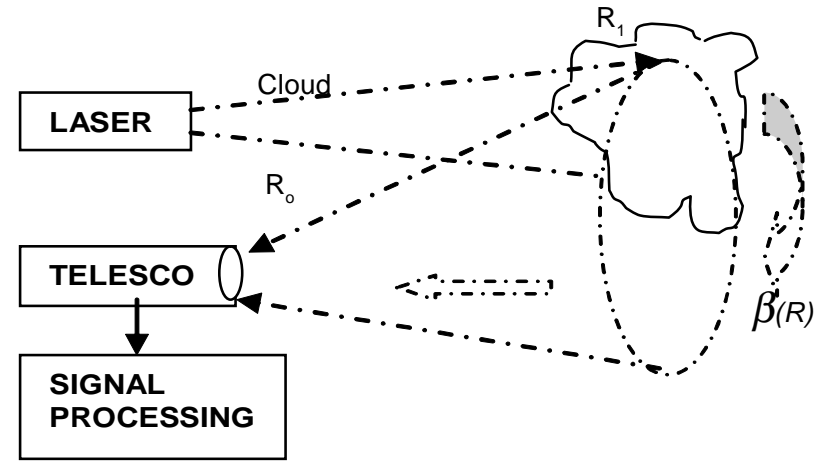

Figure 1. The simple scheme blok diagram of Lidar System

$P(R)=P_{o} A_{R} K_{o p t} \frac{G(R)}{R^{2}} \beta(R) T(R)^{2} \frac{c t_{p}}{2}+P_{b} \ldots \ldots$

Where:

$P(R)$ : is the receiver power of the return signal from a scattering volume (Watt)

$R \quad$ : is a slant range $(\mathrm{Km})$.

$\mathrm{P}_{0} \quad$ : is the transmitted peak power of laser source (Watt).

$\mathrm{K}_{\mathrm{opt}} \quad$ : is optical calibration.

$A_{R} \quad:$ is the receiver area $\left(m^{2}\right)$

$\mathrm{G}(\mathrm{R})$ : the geometrical form factor.

C : is the velocity of light $\left(\mathrm{ms}^{-1}\right)$

$t_{p} \quad:$ is the pulse width in (s)

$\hat{a}(R)$ : is the backscattering coefficient at range $R$ in $\left(\mathrm{m}^{-1} \mathrm{sr}^{-1}\right)$.

$T(R) \quad$ : is transmission to range $R$.

$\mathrm{P}_{\mathrm{b}} \quad$ : Power of background radiation.

The optical transmission is defined as: (Welton, 2002).

$T(R)=\exp \left(-\int_{0}^{R} \alpha(r) d r\right)$

Where á(r) is the extinction coefficient of aerosol and cloud in $\left(\mathrm{m}^{-1}\right)$, and $l=c t_{p} / 2$ is the transmitted pulse length, $t_{p}$ is pulse duration times, and $c$ is speed of light.

Fernald Equation. The lidar equation for two distinct classes of scatters (particular and molecular) such as in $\mathrm{Eq}$ (1) can be rewritten as follows:

$X(R)=K\left[\beta_{1}(R)+\beta_{2}(R)\right] \exp \left[-2 \int_{0}^{R}\left(\alpha_{1}(R)+\alpha_{2}(R)\right) d r\right] \ldots$

Where $K=K_{o p t} A_{R} / G(R)$ is the calibration constant of the instrument, and subscription 1,2 denoted the particular and molecular, respectively. In addition to find the solution of particle extinction coefficient, we put the lidar ratio of particle, which is defined by: (Grund, 2001).

$$
S_{1}=\frac{\alpha_{1}(R)}{\beta_{1}(R)} .
$$

Where $S_{1}$ parameter is the lidar ratio of atmospheric particle. The corresponding ratio for molecular scattering is:

$$
S_{2}=\frac{\alpha_{2}(R)}{\beta_{2}(R)} \ldots \ldots \ldots \ldots \ldots \ldots \ldots
$$

Where $\mathrm{S}_{2}$ is the lidar ratio for molecular of atmosphere. To determine the particle extinction coefficient $a_{1}(R)$, Fernald is derived in simple form as follows (Fernald, 1984) :

$$
\begin{aligned}
& \alpha_{1}(R)=-\frac{S_{1}}{S_{2}} \alpha_{2}(R)+ \\
& \frac{X(R) \exp \left[2\left(\frac{S_{1}}{S_{2}}-1\right) \int_{R}^{R_{C}} \alpha_{2}(R) d R\right]}{\frac{X\left(R_{C}\right.}{\alpha_{2}\left(R_{C}\right)+\frac{S_{1}}{S_{2}} \alpha_{2}}+2 \int_{R}^{R_{C}} X\left(R^{\prime}\right) \exp \left[2\left(\frac{S_{1}}{S_{2}}-1\right) \int_{R}^{R_{C}} \alpha_{2}(r) d r\right] d} \cdots
\end{aligned}
$$

It shows that the particle extinction coefficient $a_{1}(R)$ depends on $S_{1}$ value for particulate and $S_{2}$ for molecule. In the calculation, the value of $S_{1}$ could be assumed in range of 1 to 100 . Furthermore, the optical thickness can be derived by the integration of extinction coefficient in Eq. (6) such as follows:

$$
\tau\left(R_{\infty}\right)=\int_{R=0}^{R_{\infty}} \alpha_{1}(R) d R
$$

Where $\tau\left(R_{\infty}\right)$ is optical thickness, and $R_{\infty}$ is far-end boundary.

\section{MATERIALS AND METHOD}

In this section, the research methodology for observing the physical properties of aerosol and cloud in a tropical area is presented. The processing of lidar signal is devided by two sections, the single and multiple scattering. In this paper, we only investigate of single scattering process by means of Fernald inversion method to estimate the extinction coefficient and optical thickness at boundary layer and high altitude cloud.

The single scattering process is performed on the basis of the lidar equation. The lidar signal $P$ and range $R(\mathrm{~km})$ were the input data variables from file-data. They are multiplied by $X(R)=P(R)$. $R^{2}$. The factor $X(R)$ determines the particulate extinction coefficient $a_{1}(R)$ in Fernald calculation such as in Eq. (6). The lidar ratio for $S_{1}(R)$ parameter depends on the particulate matter of the aerosol and cloud. Moreover, the lidar ratio for 
molecules is $\mathrm{S}_{2}=8.53$. The lidar data were collected with a spatial resolution of " $R=30 \mathrm{~m}$, and maximum distance of $R_{c}=20 \mathrm{~km}$.

Furthermore, the estimation of optical thickness applied the integration of particulate extinction coefficient in Eq. (7). It may be rewritten in discrete form as follows:

$$
\tau(\mathbf{R})=\sum_{R=0}^{n} \alpha_{1}(\mathbf{R}) \Delta \mathbf{R}
$$

where $\tau(\mathbf{R})$ is the optical thickness for single scattering process.

For instrumental parameter of MPL system, the laser wavelength of $523 \mathrm{~nm}(\mathrm{Nd}: \mathrm{YLF})$ and the pulse energy of $5 \mu \mathrm{J} /$ pulse that is within the eye safety range when expanded to the diameter of $0.2 \mathrm{~m}$. The repetition frequency of the laser operationis $2.5 \mathrm{kHz}$. The receiver is a $0.2 \mathrm{~m}$ diameter Cassegrangian teleacope. In order to reduce the influence of the background noise, an optical filter with a narrow bandwidth ("l=0,2 nm) and a narrow viewing angle ( $F O V=0,1 \mathrm{mrad}$ ) The operational parameters of MPL system are shown in Table 1 such as follow:

\begin{tabular}{ll}
\multicolumn{2}{l}{ Table 1. Operational Syatem Parameters of MPL } \\
\hline System & Parameter \\
\hline Laser & Nd:YLF \\
Wavelength & $532 \mathrm{~nm}$ \\
Pulse energy & $5 \mu \mathrm{J}$ \\
Pulse Frequency & $2.5 \mathrm{KHz}$ \\
Telescope & Cassegrainian \\
Diamater Telescope & $0.2 \mathrm{~m}$ \\
Maximum FOV & $100 \mu \mathrm{rad}$ \\
Resolution & $30 \mathrm{~m}$ \\
Filter Bandwidth & $0.2 \mathrm{~nm}$ \\
Detection Quantum Efficiency & $50 \%$ \\
Background Signal & $0.2 \mathrm{~W} / \mathrm{m}^{2} / \mathrm{nm} / \mathrm{sr}$ \\
\hline
\end{tabular}

Furthermore, the lidar data processing was calculated by using the lidar signals to determine the particle extinction coefficient $\alpha_{1}(R)$ using Fernald Method in Eq (6) and optical thickness in Eq (8) The flowchart of data processing by Fernald method such as in Figure 2. In the case of analyzing data to overcome the noise problem, it was used a model of atmosphere for tropical area adopted from MODRAN 3 code of signal lidar.

\section{RESULTS AND DISCUSSION}

The single scattering process was analyzed by means of Fernald method in Eq. (6) to determine the extinction coefficient of aerosol and cloud. The Optical Thickness calculated by Eq. (8) is called the retrieved

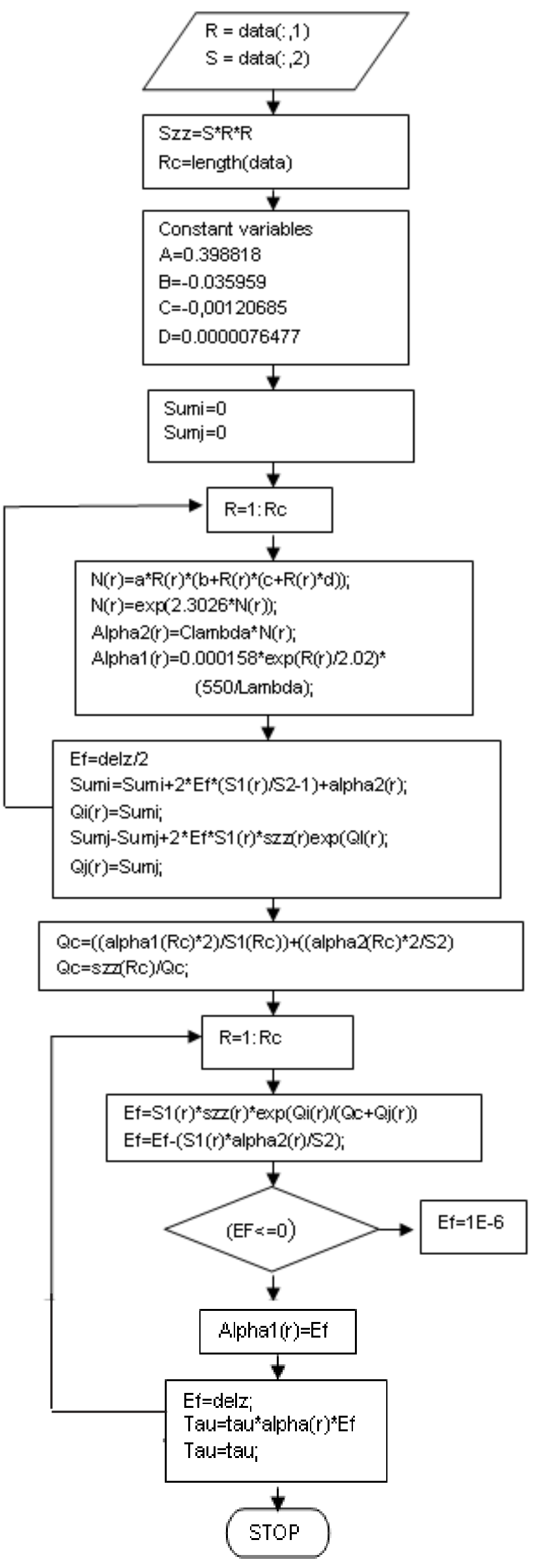

Figure 2. Flowchart of Fernald Method 
optical thickness. In this section, we used a model a layer of constant extinction coefficient and its thickness, which is varied, referred to as the optical thickness model. The retrieved optical thickness was found to saturate and reach a maximum retrieved optical thickness. Here, a simulation of optical depth is described by means of lidar data. The optical thickness model $\left(\hat{o}_{\text {mod }}\right)$ and retrieved optical thickness $\left(\hat{o}_{\text {ret }}\right)$ were simulated to estimate the maximum optical thickness. It was assumed that $\hat{o}_{\text {mod }}$ has been varied of optical thicness, and the maximum $\hat{o}_{\text {ret }}$ can be obtained from the saturation.

The results are shown in Figure 3 to Figure 7. In the case of a fixed of extinction coefficient, where the optical thickness $\hat{o}_{\text {ret }}$ retrieved from the simulated lidar signal model is plotted against the model thickness value $\hat{o}_{\text {mod }}$. The balance between the signal extinction and the detectable backscattering intensity determines the maximum optical thickness retrieved.

In Figure 3, it shows the result for the extinction coefficient $a_{1}=1 \times 10^{-3} \mathrm{~m}^{-1}$, with the maximum $\hat{o}_{\text {ret }}$ is about 3.5. In this figure, simulation curves for 50 a scope signals are depicted. The fluctuation seen for larger $\hat{o}_{\text {mod }}$ arises from the statistical behavior of the assumed noise. Similarly Figure 3 , illustrates the relationship

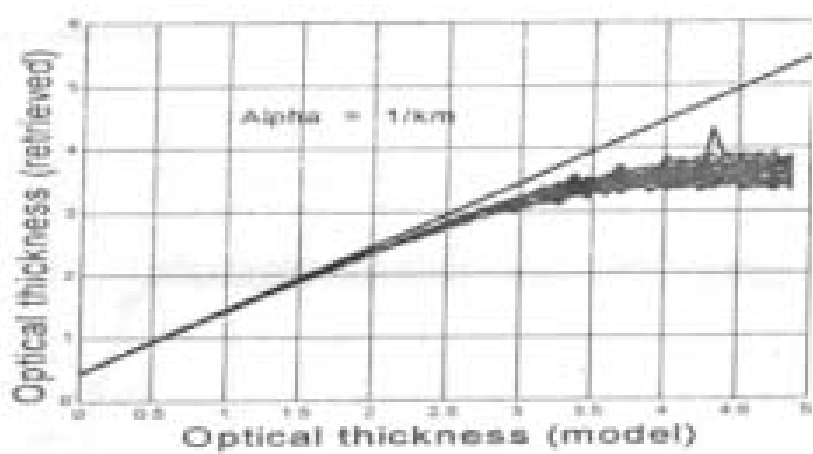

Figure 3. The estimation of optical thickness model and optical thickness retrieved in extinction coefficient (á) of $1 \times 10^{-3} \mathrm{~m}^{-1}$

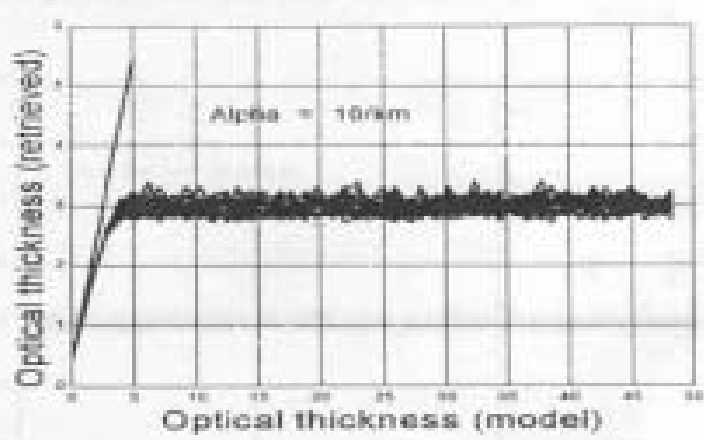

Figure 4. The estimation of optical thickness model and optical thickness retrieved in extinction coefficient (á) of $10 \times 10^{-3} \mathrm{~m}^{-1}$ between $\hat{o}_{\text {ret }}$ and $\hat{o}_{\text {mod }}$ for extinction coefficient $a_{1}=10 \times 10^{-3} \mathrm{~m}^{-1}$. The broken line shows the case where the model is recovered perfectly. Broadening of the line shows the fluctuation of the retrieved value due to fluctuation noise. The retrieved value gradually deviates from the real value. Maximum penetration depth is found around the model value of $\hat{o}_{\text {mod }}=4$, where the retrived value is about $\hat{o}_{\text {ret }}=3$ including the contribution from the background aerosol.

In Figure 5, the saturation of $\hat{o}_{\text {ret }}$ can be derived at about 2.3, at the extinction coefficien $a_{1}=20 \times 10^{-3} \mathrm{~m}^{-1}$. Furthermore, the optical thickness retrieved is around $\hat{o}_{\text {ret }}=2$, at á $a_{1}=30 \times 10^{-3} \mathrm{~m}^{-1}$ in figure 5. And $\hat{o}_{\text {ret }}$ is 1.6 at $a_{1}=40 \times 10^{-3} \mathrm{~m}^{-1}$ in Figure 7.

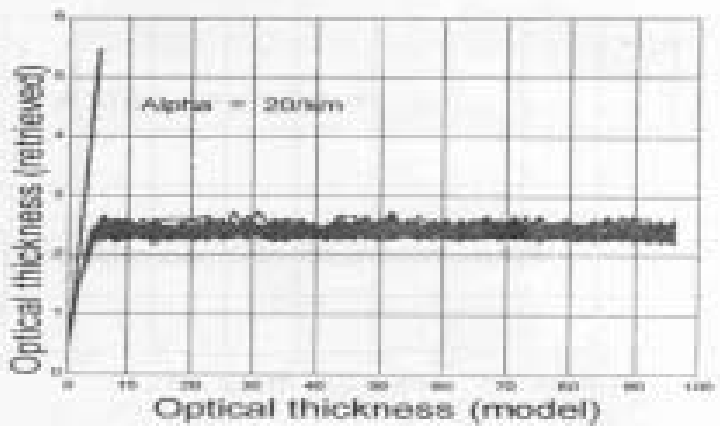

Figure 5. The estimation of optical thickness model and optical thickness retrieved in extinction coefficient (á) of $20 \times 10^{-3} \mathrm{~m}^{-1}$

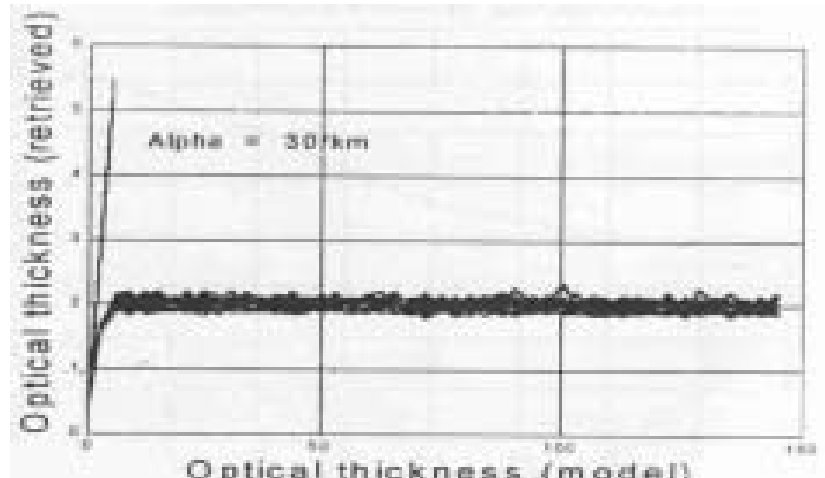

Optical thickness (model)

Figure 6. The estimation of optical thickness model and optica thickness retrieved in extinction coefficient (á) of $30 \times 10^{-3} \mathrm{~m}^{-1}$

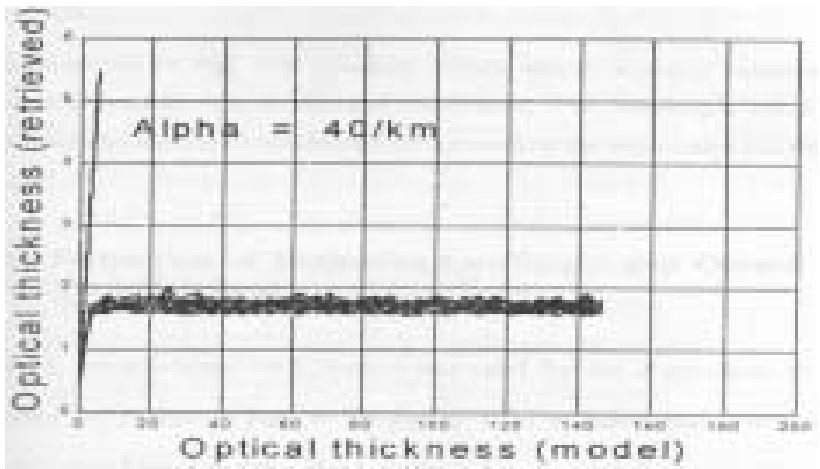

Figure 7. The estimation of optical thickness model and optical thickness retrieved in extinction coefficient (á) of $40 \times 10^{-3} \mathrm{~m}^{-1}$ 
In Figure 3 to 7, the saturation of optical thickness retrieved gradually decreases with the increase of the extinction coefficient. And the retrieved value exhibits a constant when the model value exceeds 4 .

In the second sections, we used the Micro Pulse Lidar (MPL) for observation of atmosphere. Lidar data is used by monitoring the atmosphere in a tropical area in the area of Sukhothai, Thailand on October 2007. The MPL data were processed to show the chronological change of the vertical distribution of the aerosol extinction. The MPL data were collected with a spatial resolution of $30 \mathrm{~m}$ to a distance of $20 \mathrm{~km}$. Figure 7 displays an example of 24-hour, time-height indication of the MPL signal. It is seen that the height of planetary boundary layer stayed at an altitude about $2 \mathrm{~km}$ during the observation. Figure 8 shows the timeheight image lidar data at 0:00 a.m to 24:00 p.m of one day. The low level cloud is depicted below $3 \mathrm{~km}$ high at 0:00 to 13:00 p.m. The middle level cloud is about 3 to $9 \mathrm{~km}$ high during 16:00 to 23:40 p.m. And high level cloud is above $9 \mathrm{~km}$, i.e a high altitude cloud about 9-14 km during 01:00 to 4:30 a.m, a boundary layer is also recognizable at a lower height of around $1 \mathrm{~km}$. In the present analysis, however, we concentrate on highaltitude, thick cloud from 01:00 to 4:30 a.m.

In this section, the MPL data is observed in the daytime (at 06:00 - 18:00) and the night (at 18:00 06:00). For the first, the MPL data is processed in the night to observe the lidar signal. For example, the simple of lidar signal can be seen in Figure 9. A high altitude cloud is illustrated in 11 to $13 \mathrm{~km}$ high at 01:00:40 a.m. Maximum of original signal is $5.3 \mathrm{PhE} / \mu \mathrm{sec} \mathrm{km}^{2}$. Here, $\mathrm{PhE}$ is photon energy in Joule. The simple estimation of extinction coefficient was calculated at high altitude cloud such as in Figure 10 at 01:00:40 a.m.

A maximum extinction coefficient is found at $a_{1}=6 \times 10^{-4} \mathrm{~m}^{-1}$, and at boundary layer of aerosol is $a_{1}=1.9 \times 10^{-4} \mathrm{~m}^{-1}$ in $1 \mathrm{~km}$ high. Further more the optical

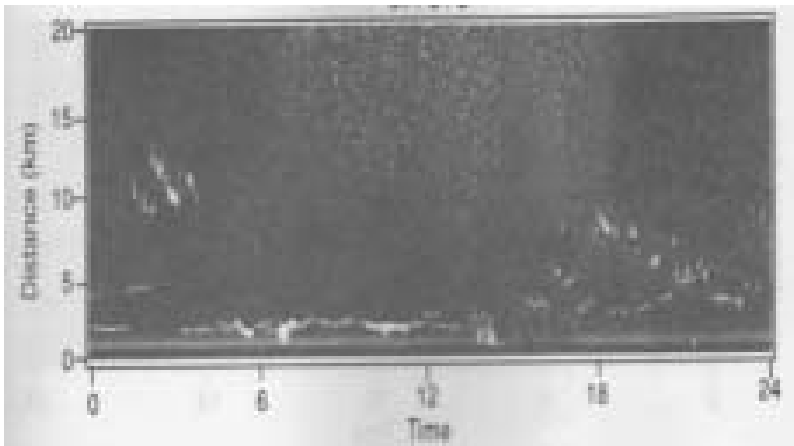

Figure 8. Simple of Time-height image of MPL signal
Retrieved Optical Thickness for analyzing

11 thickness is calculated for several cases such as in figure 10. A maximum optical thickness $(\hat{o}=2.60)$ is found for the data 01:00:40 a.m, with an extinction coefficient of about $\mathrm{a}_{1}=6 \times 10^{-4} \mathrm{~m}^{-1}$. A minimum value (ô $=0.50$ ) is obtained at 01:11:17 a.m. Then, the maximum optical thickness of sky-radiometer as displayed on monitor was 2.55, as reference data. Our result that was displayed in Figure 11 indicates a reasonable with the presented simulation diagram in Figure 3 to Figure 7. The saturation behavior of the cloud optical thickness, in principle, brought about by the penetration and attenuation processes of the laser light in the cloud. Thus, it does not yield direct information on the optical thickness properties of tropical clouds.

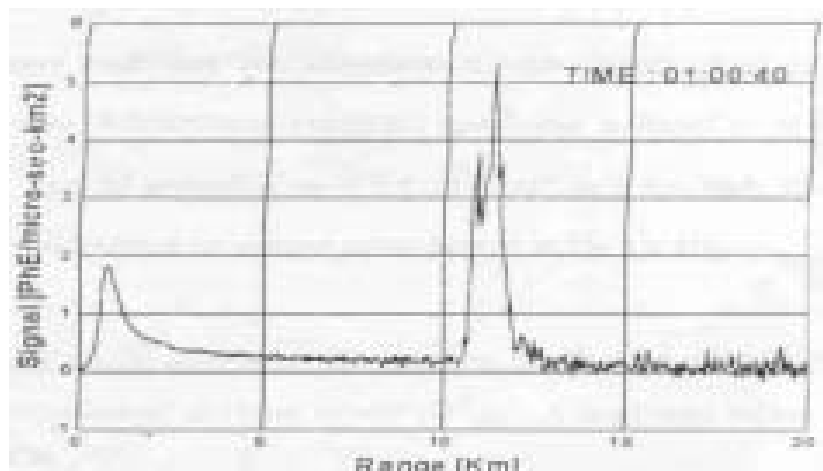

Figure 9. The lidar signal in high-level cloud is shown at the time 01:00:40 a.m

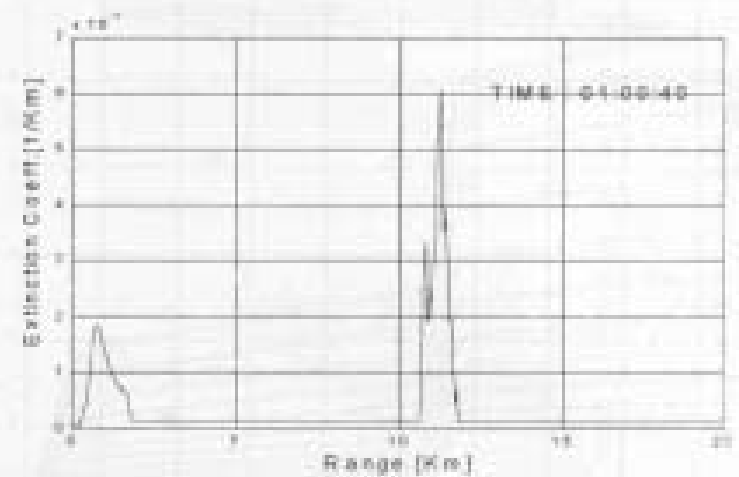

Figure 10. The extinction coefficient of high altitude clouds 01:00:40 a.m

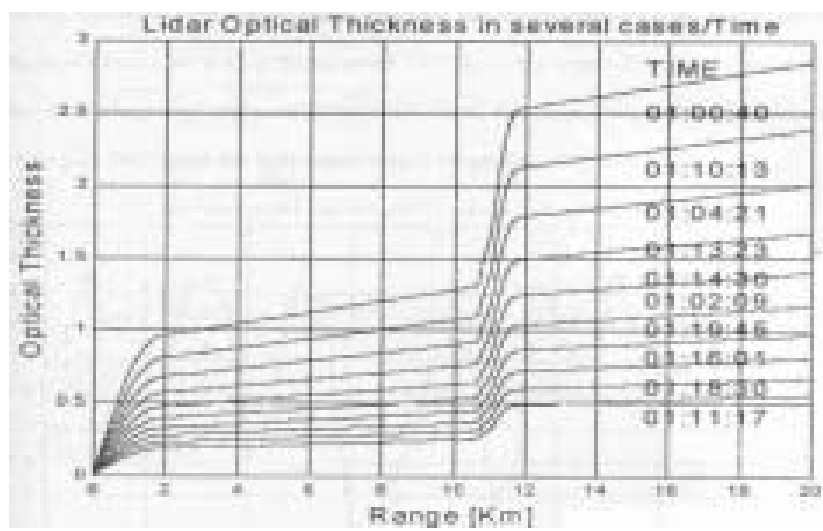

Figure 11. The optical thickness in any cases at high altitude cloud 
Nevertless, this behavior is important to interpreter the apparent variation of cloud thickness, since in some occasion; the cloud thickness hinders direct detection of the cloud top from the lidar signal. It is also noted that concerning the saturation effect, the maximum optical thickness is also depends on the parameter of the lidar system.

Furthermore, the estimation of lidar optical thickness at the day time (06:00 - 18:00) is shown in Figure 12 on Oktober 2007. Here, the optical thickness from sky-radiometer (wavelength of $500 \mathrm{~nm}$ ) is applied as reference data to compare the results of lidar estimation (at wavelength of $523 \mathrm{~nm}$ ).

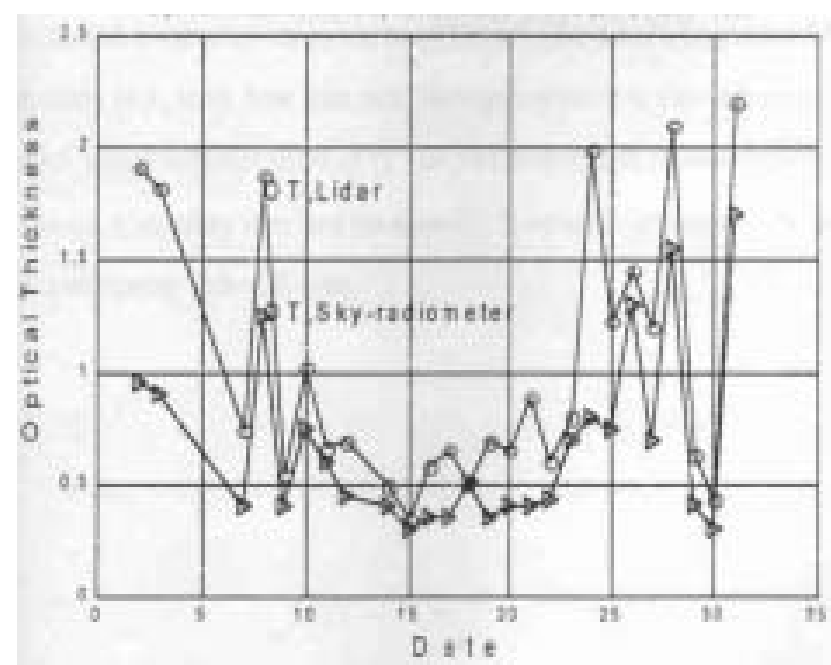

Figure 12. The maximum optical thicness of lidar (circle), and sky-radiometer data(triangular) in every day on October 2007

The optical thickness of lidar and sky-radiometer are noted by circle and triangular, respectively, such as in Figure 12. We derived one point of maximum optical thickness in every day to plot in curve on october 2007 at the time of $06.00-18.00$. The maximum of lidar optical thickness and sky-radiometer in october are 2.25 and 1.7. These show that the value of lidar and sky-radiometer are different. It is caused by the differently wavelength. The lidar wavelength is $523 \mathrm{~nm}$ and sky-radiometer of $500 \mathrm{~nm}$. The value of lidar optical thickness is bigger then sky-radiometer. We noted that receiver of sky-radiometer limited by the sun intensity and the weather condition. However, lidar is independent of the sun intensity. It may be operated on the time and at night. This is the limitation of the sky-radiometer does not apply to lidar.

\section{CONCLUSIONS}

The micro pulse lidar (MPL) was applied to observe the aerosol and cloud properties of the atmosphere in a tropical area. It was important and simple applications of remote sensing for monitoring the atmosphere.

The retrieved optical thickness was simulated by using Fernald inversion method. The results were indicated by the saturation, the retrieved optical thickness decreases whith the increase of the extinction coefficient.

The optical thickness properties of aerosol and cloud were analyzed by using MPL lidar operated at $523 \mathrm{~nm}$. There were compared by the optical thickness from sky radiometer operated at $500 \mathrm{~nm}$. This result shows that the radiometer optical thickness is a good estimation of the retrieved lidar optical thickness.

\section{ACKNOWLEDGEMENTS}

The writer is deeply indepted to Prof. Nobuo Takeuchi from Center for Environmental Remote Sensing (CEReS), Chiba University, Japan, for providing lidar data from Sukhothai, Thailand in a tropical area.

\section{REFERRENCES}

Dewang, S. \& Takeuchi, N. 2000 The Observation of Optical Thickness in 3 level height of clouds by using micro pulse lidar IECI, Japan.

Fernald, F.G. 1984 Analisis of atmosphere lidar Observations: some comments: Appl. Opt 23.

Grund, Christian J., Banta, Robert M., George, Joanne, L. \& Howell, James, N. 2001 High-resolution Doppler lidar for boundary layer and cloud research: Journal of Atmospheric and Oceanic Technology.

Lottman, B.T., Frehlich, R.G., Hannon, S.M. \& Henderson, S.W. 2001 Evaluation of vertical winds near and inside a cloud deck using coherent Doppler lidar, J. Atmos. Oceanic Technol.

Niranjan, K., Madhavan, B.L. \& Sreekanth, V. 2007, Micro pulse lidar observation of high altitude aerosol layers at Visakhapatnam located on the east coast of India, Geophys. Res. Lett 34.

Shiina, T., Yoshida, K., Ito, M. \& Okamura, Y. 2005 In-line type micropulse lidar with an annular beam: experiment, Appl. Opt 44: 7407-7413.

Welton, E.J. \& Campbell, J.R. 2002. Micro-pulse Lidar Signals: uncertainty analysis. J. Atmos. Oceanic Technol. 